\title{
Modal Resonant Frequencies and Radiation Quality Factors of Microstrip Antennas
}

\author{
Jan Eichler, Pavel Hazdra, Miloslav Capek, and Milos Mazanek \\ Department of Electromagnetic Field, Faculty of Electrical Engineering, Czech Technical University in Prague, Technická 2, \\ 166 27, Prague, Czech Republic \\ Correspondence should be addressed to Pavel Hazdra, hazdrap@fel.cvut.cz
}

Received 9 August 2011; Revised 10 January 2012; Accepted 13 January 2012

Academic Editor: Charles Bunting

Copyright ( 92012 Jan Eichler et al. This is an open access article distributed under the Creative Commons Attribution License, which permits unrestricted use, distribution, and reproduction in any medium, provided the original work is properly cited.

The chosen rectangular and fractal microstrip patch antennas above an infinite ground plane are analyzed by the theory of characteristic modes. The resonant frequencies and radiation $Q$ are evaluated. A novel method by Vandenbosch for rigorous evaluation of the radiation $Q$ is employed for modal currents on a Rao-Wilton-Glisson (RWG) mesh. It is found that the resonant frequency of a rectangular patch antenna with a dominant mode presents quite complicated behaviour including having a minimum at a specific height. Similarly, as predicted from the simple wire model, the radiation $Q$ exhibits a minimum too. It is observed that the presence of out-of-phase currents flowing along the patch antenna leads to a significant increase of the $Q$ factor.

\section{Introduction}

Evaluation of the basic properties of microstrip patch antennas (MPA) has been numerously discussed in literature, see, for example, [1-3]. The two main MPA attributes are resonant frequency (or frequencies but we will deal mostly with the dominant mode) and the radiation $Q$ factor. So far, only approximate results and semianalytic equations have been published. To our knowledge, this is the first time that these important characteristics have been studied in a rigorous way. The antennas are treated by using a modal approach (hence we do not a priori consider any feeding to be connected), namely, by the theory of characteristic modes (TCM), [4, 5]. Evaluation of the radiation $Q$ is performed both by the TCM from the eigenvalues slope and by novel rigorous equations derived by Vandenbosch [6] and Vandenbosch and Volski [7].

\section{The Theory of Characteristic Modes}

For completeness, let us formulate the basics of the characteristic modes for perfectly conducting bodies of area $S$. The scattered field $\mathbf{E}^{s}$ is related to the electric surface currents $\mathbf{J}$ by the electric field integral equation (EFIE) [8]

$$
\left[L(\mathbf{J})-\mathbf{E}^{i}\right]_{\tan }=0 \text {. }
$$

Equation (1) is usually treated within the method of moments (MoMs) [8] framework and, due to the structure discretization, the $\mathbf{L}$ operator is known as the "complex impedance matrix" $[Z]=[R]+j[X]$.

Then the associated Euler's equation to be solved is

$$
X \mathbf{J}_{n}=\lambda_{n} R \mathbf{J}_{n}
$$

Equation (2) is a standard weighted eigenvalue equation leading to a set of real characteristic eigencurrents $\mathbf{J}_{n}$ and associated eigenvalues $\lambda_{n}$. Properties of eigenvalues are described in [9], at this moment it is important to note that $\lambda_{n}$ reflects the amount of net reactive power (thus $\lambda_{n}=$ 0 means resonance). Instead of eigenvalues, the so-called characteristic angles $\alpha_{n}$ are introduced to show more visible behavior with frequency [9]. Characteristic currents form a complete orthogonal set, and hence the total current on a conducting body may be expressed as a linear combination of these mode currents [10].

2.1. Implementation of the Characteristic Modes Theory. Implementation of the modal decomposition process has been done in the MATLAB [11] environment using Makarov EFIE codes [12] with the RWG basis functions [13]. This usage is restricted to arbitrary 3D PEC structures with air 
dielectrics. Our developed TCM tool [14] has the following main advantages:

(i) Comsol Multiphysics [15]/MATLAB’s PDE TooLbox mesh import,

(ii) Optional Green's function for infinite ground plane simulations,

(iii) Single solver/multicore solver/distributed solver (within a computer network with installed MATLAB).

\section{The Radiation $Q$ Factor}

In [6] a novel theory able to rigorously calculate radiated power and stored energies directly from currents flowing along the antenna has been presented. The radiation $Q$ factor is then readily evaluated by the definition [16]:

$$
Q=2 \omega \frac{\max \left(\widetilde{W}_{m}, \widetilde{W}_{e}\right)}{P_{r}} .
$$

The equations for radiated power $P_{r}$ and stored electric and magnetic energies $\widetilde{W}_{e}, \widetilde{W}_{m}$ are

$$
\begin{aligned}
P_{r}=\left(\frac{1}{8 \pi \omega \varepsilon_{0}}\right) \int_{\Omega 1} \int_{\Omega 2}[ & \left.k^{2} \mathbf{J}\left(\mathbf{r}_{1}\right) \mathbf{J}\left(\mathbf{r}_{2}\right)-\nabla \cdot \mathbf{J}\left(\mathbf{r}_{1}\right) \nabla \cdot \mathbf{J}\left(\mathbf{r}_{2}\right)\right] \\
& \times \frac{\sin \left(k r_{21}\right)}{r_{21}} d \Omega 1 d \Omega 2 \\
\widetilde{W}_{e} & =\frac{1}{16 \pi \omega^{2} \varepsilon_{0}}\left(I_{e}-I_{R}\right) \\
\widetilde{W}_{m} & =\frac{1}{16 \pi \omega^{2} \varepsilon_{0}}\left(I_{m}-I_{R}\right)
\end{aligned}
$$

where

$$
\begin{gathered}
I_{R}=\frac{k}{2} \int_{\Omega 1} \int_{\Omega 2}\left[k^{2} \mathbf{J}\left(\mathbf{r}_{1}\right) \mathbf{J}\left(\mathbf{r}_{2}\right)-\nabla \cdot \mathbf{J}\left(\mathbf{r}_{1}\right) \nabla \cdot \mathbf{J}\left(\mathbf{r}_{2}\right)\right] \\
\times \sin \left(k r_{21}\right) d \Omega 1 d \Omega 2, \\
I_{e}=\int_{\Omega 1} \int_{\Omega 2} \nabla \cdot \mathbf{J}\left(\mathbf{r}_{1}\right) \nabla \cdot \mathbf{J}\left(\mathbf{r}_{2}\right) \frac{\cos \left(k r_{21}\right)}{r_{21}} d \Omega 1 d \Omega 2, \\
I_{m}=k^{2} \int_{\Omega 1} \int_{\Omega 2} \mathbf{J}\left(\mathbf{r}_{1}\right) \mathbf{J}\left(\mathbf{r}_{2}\right) \frac{\cos \left(k r_{21}\right)}{r_{21}} d \Omega 1 d \Omega 2,
\end{gathered}
$$

where $k$ is a free-space wavenumber, $\mathbf{J}$ is the surface current density, and $r_{21}$ is the distance between interacting current elements. The tilde denotes that the radiation contribution $I_{R}$ has been subtracted from the stored energies at every point in space [17]. It is assumed that the currents are flowing in a vacuum.

3.1. The Modal Radiation $Q$ Factor. The modal radiation $Q$ factor may be evaluated from the slope of modal eigenvalues [18]:

$$
Q_{\text {eig }}=\frac{\omega_{0}}{2} \frac{d \lambda}{d \omega} \text {. }
$$

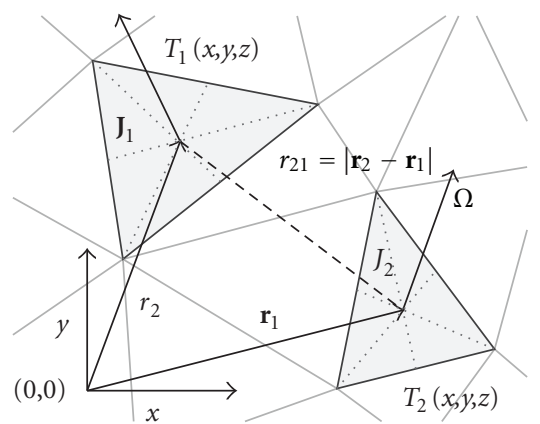

FIGURE 1: Distance between nonoverlapping current elements [23].

In [18], (10) is supposed to be an approximation of the radiation $Q$, but in resonance it is actually exact.

Since characteristic modes are normalized to radiate unit power $P_{r}=1$ [4], (3) reduces to

$$
Q=2 \omega \max \left(\widetilde{W}_{e}, \widetilde{W}_{m}\right) .
$$

For parallel or series RLC circuit (hence, for one mode), the “impedance $Q z$ ” equals the exact "current $Q$ ” [6]:

$$
Q=Q_{Z}=\frac{\omega_{0}}{2}\left|\frac{\partial Z}{\partial \omega}\right|=\frac{\omega_{0}}{2}\left|\frac{\partial R}{\partial \omega}+\frac{\partial X}{\partial \omega}\right| .
$$

Inserting

$$
Z=R+j X=\frac{1}{\left|I^{2}\right|}\left[P_{r}+j 2 \omega\left(\widetilde{W}_{m}-\widetilde{W}_{e}\right)\right]
$$

valid for lossless antennas [19] and using the fact that $P_{r}=1$, (12) results in

$$
\begin{aligned}
Q & =Q_{X}=\frac{\omega_{0}}{2}\left|\frac{\partial X}{\partial \omega}\right|=\frac{\omega_{0}}{2} \frac{\partial}{\partial \omega}\left[2 \omega\left(\widetilde{W}_{m}-\widetilde{W}_{e}\right)\right] \\
& =\frac{\omega_{0}}{2} \frac{\partial \lambda}{\partial \omega}=Q_{\text {eig }},
\end{aligned}
$$

providing that

$$
\lambda=2 \omega\left(\widetilde{W}_{m}-\widetilde{W}_{e}\right) .
$$

It is therefore concluded that the modal $Q_{\text {eig }}$ equals the $Q_{X}$ by definition, and it can be proven (using the reactance theorem $[20,21])$ that in resonance it also equals the radiation $Q$ defined from energies by (11).

3.2. Software Implementation. The above equations were implemented in MATLAB for the RWG triangular mesh where two different interaction situations occur:

(a) Distant Elements. When the triangular elements are not overlapping, current density on triangles may be simply approximated as point sources located at the centre of triangles [22], see Figure 1. No actual integration is then needed. This centroid approach is very fast with satisfactory accuracy as will be shown later (however it may fail for patches located very close to the ground plane). 


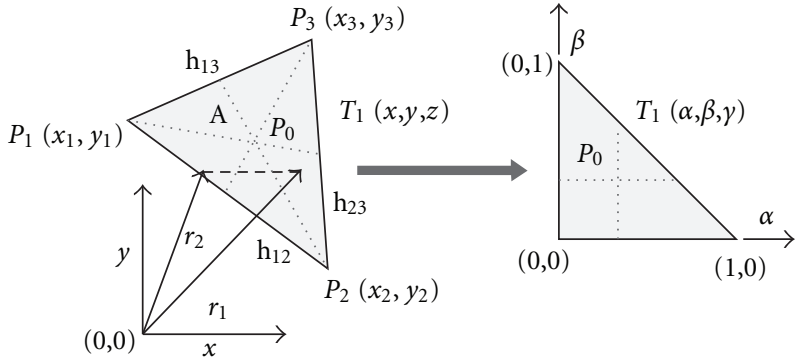

(a)

(b)

Figure 2: Self-term evaluation. (a) Original problem, (b) simplex coordinates transformation [23].

(b) Overlapping (Self) Elements. As known from the method of moments, the so-called "self" contributions are of great importance when dealing with calculations on discrete elements (meshes).

Here, the self-interaction occurs when two triangles are overlapping each other. Due to the behavior of integral kernels, only rapidly varying term $\cos \left(k r_{21}\right) / r_{21}$ has to be carefully treated. Since $k_{0} R_{21} \rightarrow 0\left(R_{21}\right.$ being the longest side of the triangle $T$ ) is satisfied, one needs only to use the first term in the Taylor series expansion. The dominant singular static part is $1 / r_{21}$ and the integral to be worked out is

$$
I=\int_{T} \int_{T^{\prime}} \frac{1}{\sqrt{\left(x-x^{\prime}\right)^{2}+\left(y-y^{\prime}\right)^{2}}} d x d y d x^{\prime} \mathrm{d} y^{\prime},
$$

where $T=T^{\prime}$ is a triangular area. Using simplex coordinates transformation (Figure 2), the result is $[23,24]$

$$
I=-\frac{4}{3} A^{2}\left[\frac{\ln \left(1-2 h_{12} / L\right)}{h_{12}}+\frac{\ln \left(1-2 h_{13} / L\right)}{h_{13}}+\frac{\ln \left(1-2 h_{23} / L\right)}{h_{23}}\right] \text {, }
$$

where $A$ is the triangle area, $h_{i j}$ are the edge lengths (see Figure 2), and $L$ is the perimeter of the triangle.

\section{Applications: Rectangular Patch Antenna}

Let us first concentrate on a rectangular patch antenna of dimensions $L=50 \mathrm{~mm}$ and $W=30 \mathrm{~mm}$ (further noted as $\mathrm{R} 50 \times 30)$ placed in air at a height $H$ above an infinite ground plane. Only the dominant $\mathrm{TM}_{01}$ mode will be studied. The reason for choosing a patch with $L / W \neq 1$ is that we do not have to deal with degenerated modes.

Using the image theory, the radiator in the $X Y$ plane at height $z=H$ above an infinite electric ground plane is modelled as two patches separated by $2 H$. The total number of triangular elements is 676. In the TCM analyser, a proper out-of-phase mode is selected (Figure 3).

The resonant frequency of the dominant mode is shown as a function of height $H$, see Figure 4 . It has been evaluated from a modal resonant condition for eigenvalues $\lambda=$ $2 \omega\left(\widetilde{W}_{m}-\widetilde{W}_{e}\right)=0$ employing an adaptive frequency sweep for each height. The behaviour is quite peculiar, especially for greater heights. For low heights $(H<10 \mathrm{~mm}$ or

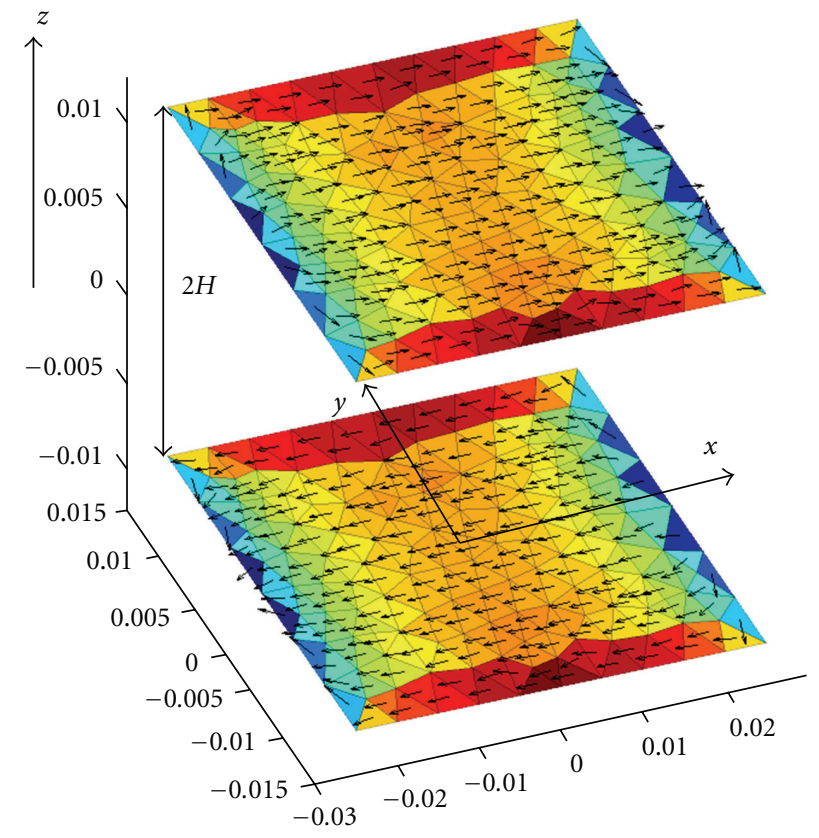

Figure 3: Model of MPA above infinite ground plane for $H=$ $10 \mathrm{~mm}$, dominant mode $\mathrm{TM}_{01}$ shown.

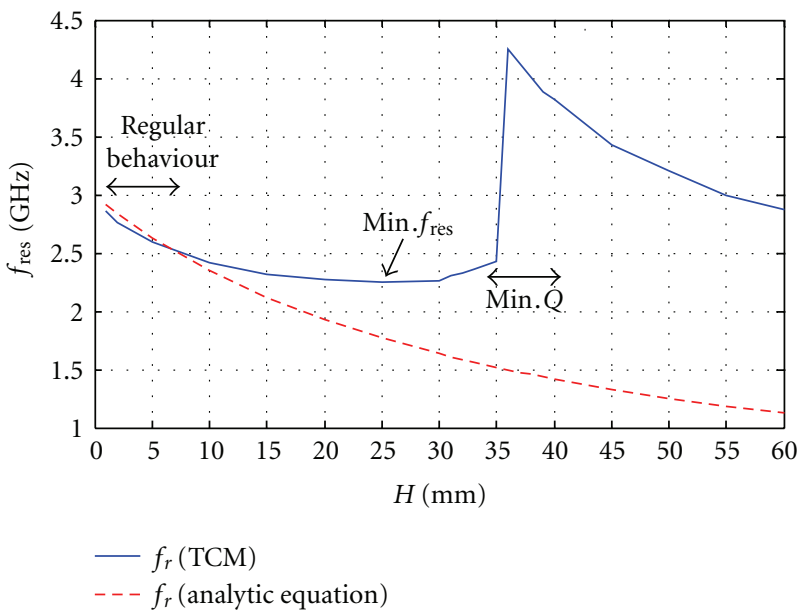

Figure 4: $\mathrm{R} 50 \times 30$ resonant frequency of the dominant $\mathrm{TM}_{01}$ mode. The dashed red curve is a quasianalytical equation from [1].

$\left.H / \lambda_{\text {res }}<0.08\right)$, the resonant frequency decreases "regularly," and quasianalytical formulas (see, e.g., [1, 3]) based on the fringing field concept are valid below this range. For $H \cong 25 \mathrm{~mm}\left(H / \lambda_{\text {res }} \cong 0.188\right)$ there is absolute minimum of the $\mathrm{TM}_{01}$ resonant frequency. Further on, the resonant frequency rises to reach its maximum for $H \cong 40 \mathrm{~mm}$ $\left(H / \lambda_{\text {res }} \cong 0.51\right)$. Around this specific height the patch also shows the minimum of the radiation $Q$. The above described process repeats periodically. It is yet unclear to the authors as what is the physical background to the resonant frequency discontinuity around $H / \lambda_{\text {res }} \cong 0.5$.

The terms $2 \omega \widetilde{W}_{m}, 2 \omega \widetilde{W}_{e}$, and $2 \omega\left(\widetilde{W}_{m}-\widetilde{W}_{e}\right)$ obtained from (5)-(9) and eigenvalues $\lambda$ are plotted at Figure 5 for 


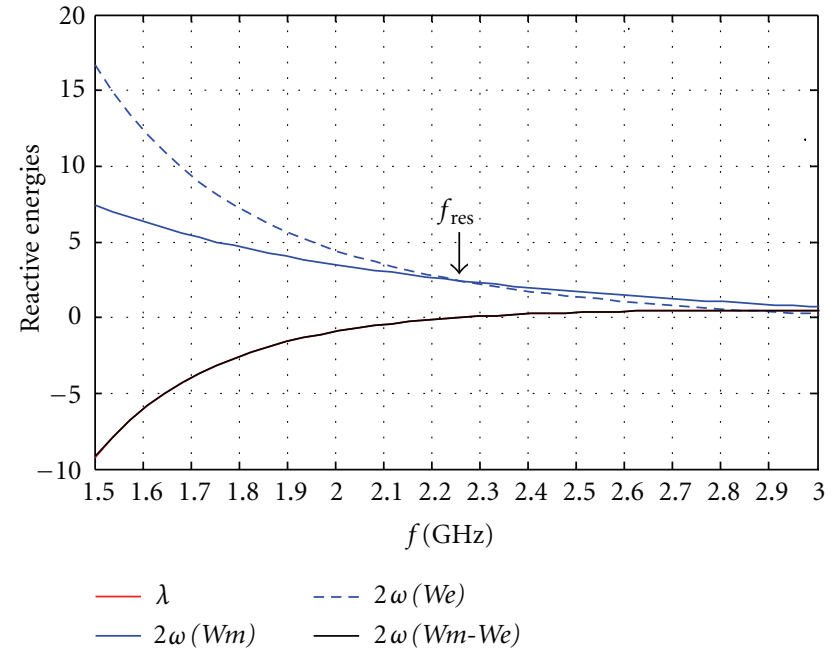

FIGURE 5: Reactive energies and their differences for an R50 $\times 30$ patch at height of $25 \mathrm{~mm}$.

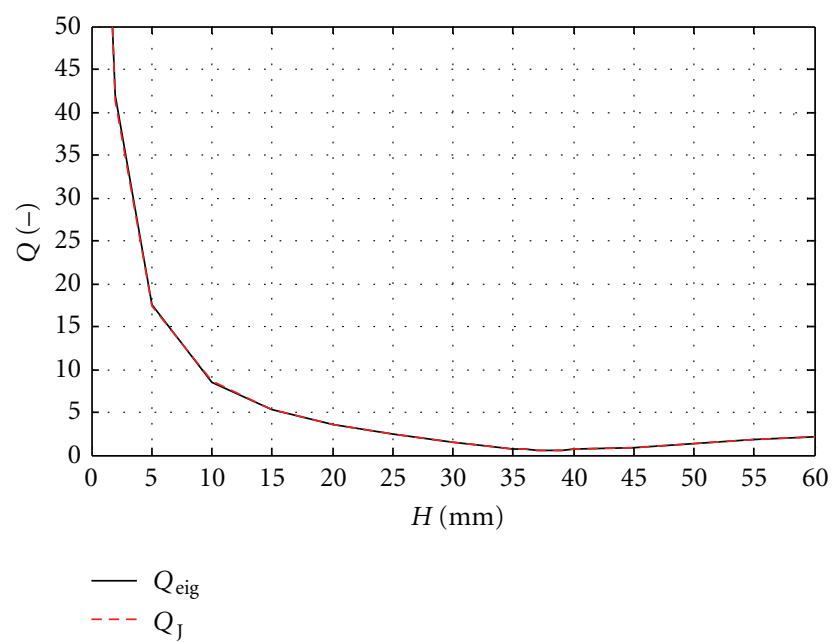

FIgURE 6: The radiation $Q$ for dominant mode of an $R 50 \times 30$ patch as a function of height $H$.

$H=25 \mathrm{~mm}$ as a function of frequency. There is excellent agreement between the difference in stored energies and the eigenvalues, both obtained in a completely different manner.

There is also very good agreement between the exact $Q_{J}$ and $Q_{\text {eig }}$ confirming the validity of the proposed algorithm via (14), see Figure 6. Note that $Q_{\text {eig }}$ in (10) does not require the currents to be calculated on the structure while $Q_{J}$ is evaluated in a rigorous way from modal currents (11).

From Figure 6 it is seen that the radiation $Q$ has a minimum for a specific height. It is deduced that the reason lies in the cancelling of the radiated power between the two out-of-phase currents. Similar behaviour has been observed in the case of two half-wave thin-wire dipoles with opposite sinusoidal currents, separated by $d=2 H$, see [25] for details. Actually these two out-of-phase dipoles may serve as a very simple model for a patch antenna with a dominant mode. When the dipoles are reduced to elementary (Hertzian) ones, an approximate analytical solution is available and in [25] we showed that the $Q$ is led by the function

$$
f_{Q}(H) \cong \frac{2 H}{k 2 H-\sin (k 2 H)} .
$$

After deriving (18), the condition is worked-out

$$
\tan (k 2 H)=k 2 H,
$$

and the first nontrivial root of (19) could be approximated as [25]

$$
\left(\frac{H}{\lambda}\right)_{\min } \cong \frac{3}{8}-\frac{1}{6 \pi^{2}}=0.358 .
$$

For sinusoidal currents on dipoles the minimum (evaluated numerically) occurs for $H=0.36 \lambda$.

The minimum of the patch under study is obtained at $H \cong 0.4 \lambda$, a value that is remarkably close to the simple dipole model.

4.1. Algorithm Convergence. Since no other methods for calculating modal $Q$ are available, $Q_{\text {eig }}$ is taken as a reference, and the relative error percentage is defined as:

$$
\text { relative error }=\frac{\left|Q_{J}-Q_{\mathrm{eig}}\right|}{Q_{\mathrm{eig}}} \cdot 100,
$$

where $Q_{J}$ is calculated from the currents using (11). Four different heights $H$ were chosen, $H=1 \mathrm{~mm}(0.01 \lambda), H=$ $2 \mathrm{~mm}(0.0185 \lambda), H=10 \mathrm{~mm}(0.0803 \lambda)$, and $H=20 \mathrm{~mm}$ $(0.151 \lambda)$, and the relative error was evaluated as a function of total triangular elements (including the mirror), see Figure 7. All quality factors were evaluated at the resonant frequency of the dominant mode for the $\mathrm{R} 50 \times 30$ patch. As discussed earlier, the centroid approximation became more inaccurate with low heights $H$. However, even for the lowest analyzed value $H=0.01 \lambda$, the relative error is in the order of a few percent for reasonable mesh density (hundreds of elements). Further improvements to the integration routine are considered for the future.

4.2. Fractional Bandwidth of the $R 50 \times 30$ Patch Antenna. It is known that the fractional bandwidth (FBW) is related to the unloaded $Q$ factor and the desired matching VSWR level. For VSWR $<s$ we have [26]

$$
\mathrm{FBW} \cong \frac{s-1}{Q \sqrt{s}}[\%] .
$$

Using a full-wave simulator CST-MWS [27], an R50 × 30 patch has been simulated and the FBW $\mathrm{CST}_{\text {for }} \mathrm{VSWR}<2$ was calculated as:

$$
\mathrm{FBW}_{\mathrm{CST}}=\frac{f_{2}-f_{1}}{f_{0}},
$$

where $f_{2}$ and $f_{1}$ are margins for VSWR $<2$ and $f_{0}$ is the centre frequency. Only very low heights were studied since we used a simple probe feed which introduces an inductance component to the total input impedance. The comparison in Figure 8 shows good agreement of both fractional bandwidths. 


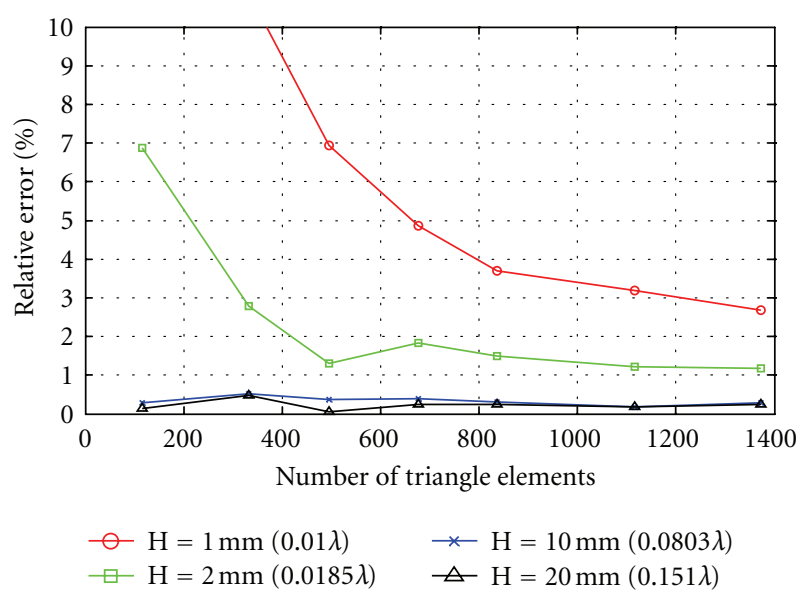

FIgURE 7: Relative error of the $Q$ factor as a function of triangular elements (mesh density).

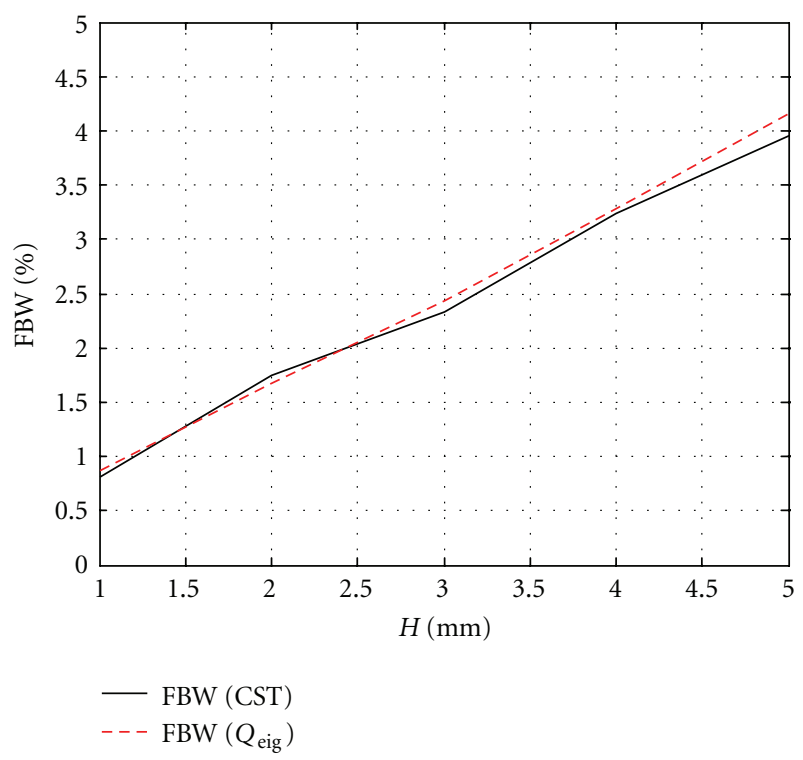

FIgURe 8: Fractional bandwidth FBW $($ VSWR $<2$ ) for a R50 × 30 patch.

\section{Applications: Fractal Antennas}

In this section, a bit more complex structures will be studied. The first one (the "Self Affine U" fractal, SAU), has been described in [28] and further analyzed in [29]. This kind of radiating motif is employed as a dual-band radiator with mutually orthogonal radiation patterns at both bands. Therefore we are analyzing the first two modes, where the currents are orthogonal. These are depicted in Figures 9 and 10 for first (SAU1) and second (SAU2) fractal iteration, respectively. The current of the first (lower) mode $\mathbf{J}_{1}$ has two out-of-phase components (see Figure 11 for schematic current paths) while the second mode comprises inphase currents only. As we know from previous studies, opposite currents contribute to a rapid increase of the radiation $Q$, and it is expected that $\mathbf{J}_{1}$ will have a much higher $Q$ than $\mathbf{J}_{2}$.

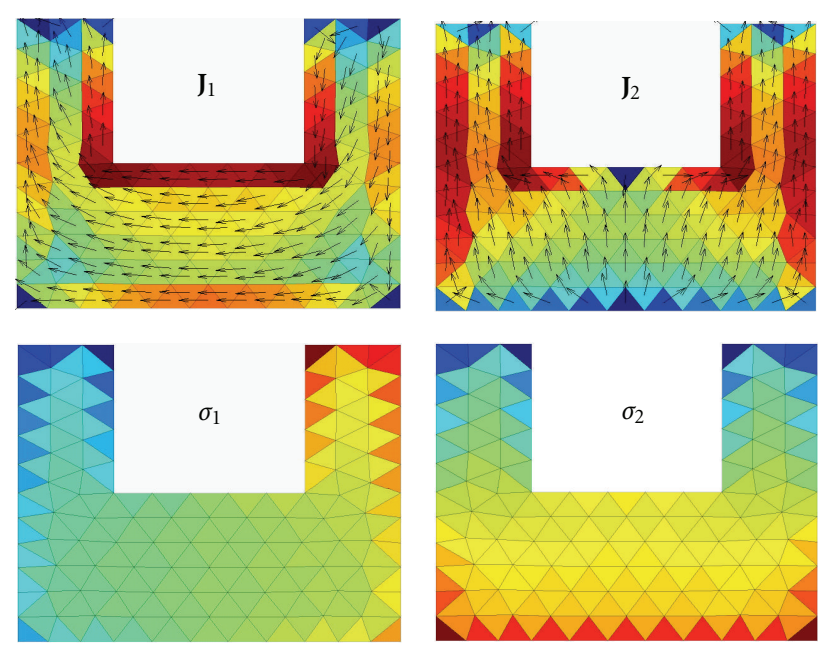

FIGURE 9: The first two characteristic modes (currents and charges) for the SAU1 structure.

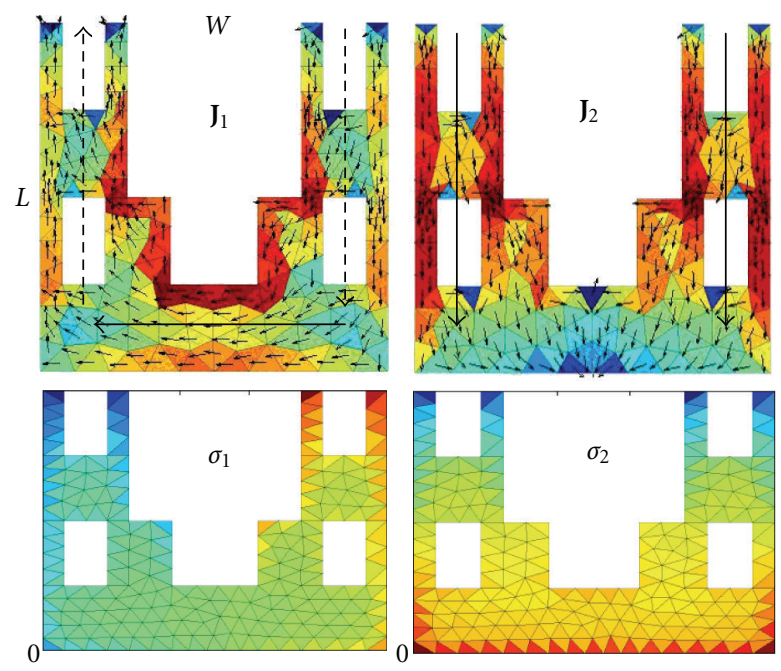

Figure 10: The first two characteristic modes (currents and charges) for the SAU2 structure.

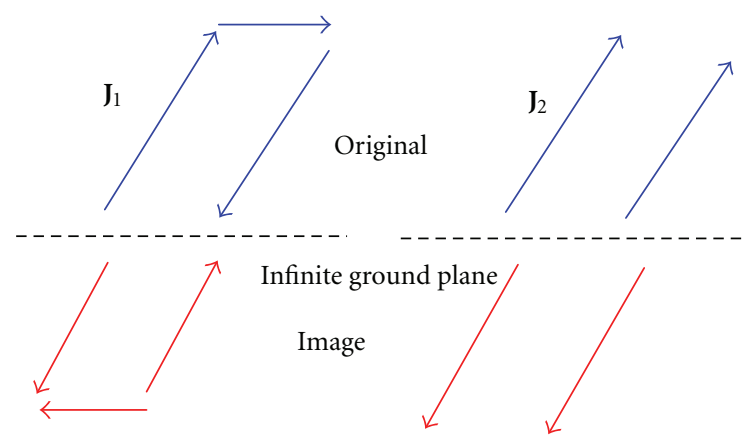

FIGURE 11: The main current paths for the first two modes of the SAU1/2 structure. 


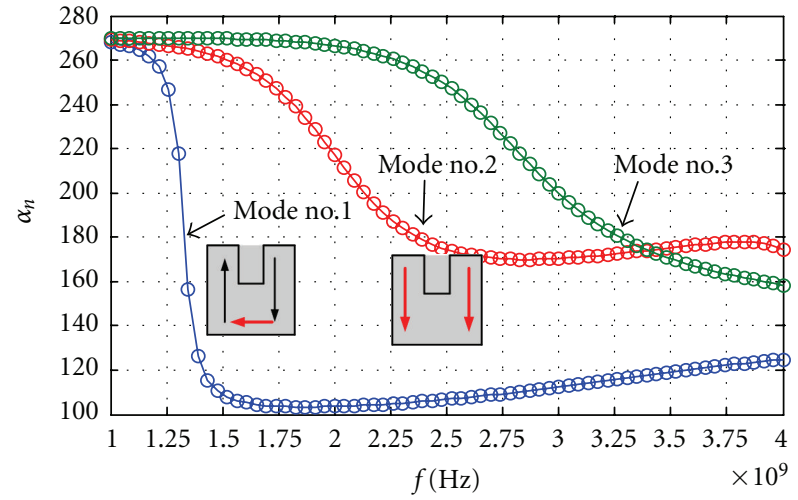

(a)

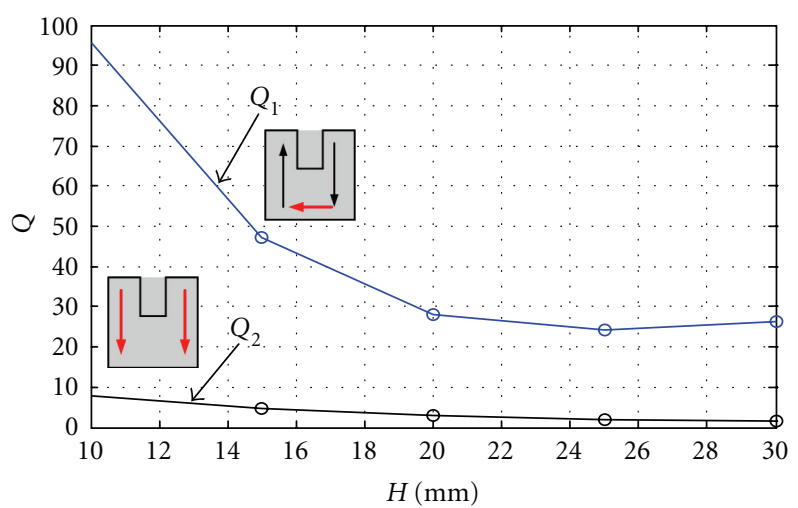

(b)

FIgURE 12: Characteristic angles (left) and radiation $Q$ for the SAU2.

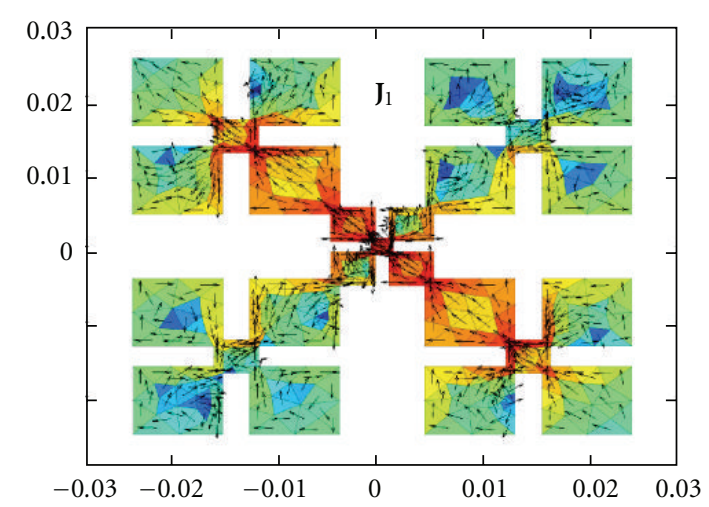

(a)

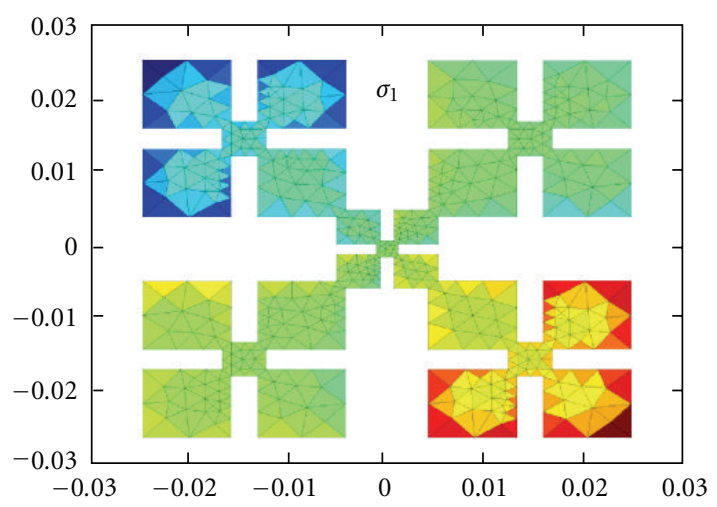

(c)

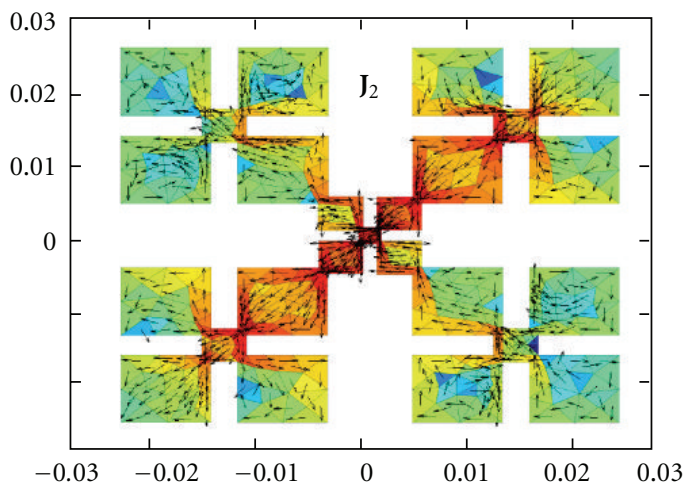

(b)

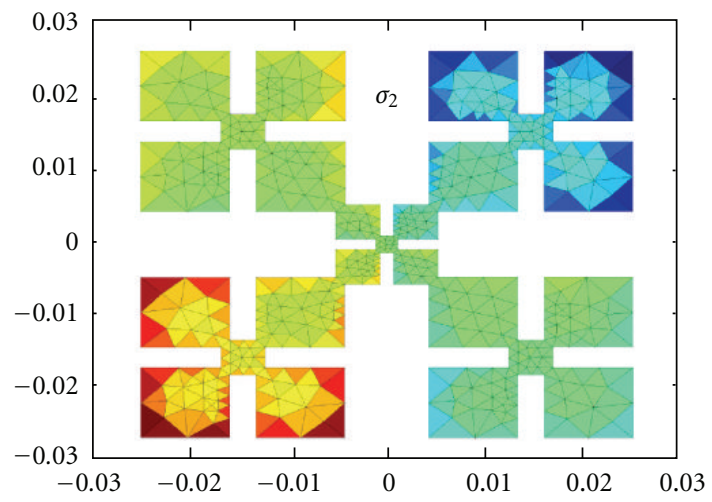

(d)

Figure 13: Degenerated dominant mode $\mathbf{J}_{1}$, and $\mathbf{J}_{2}$ of the FCL2 antenna (currents and charges).

Figure 11 presents a very simple concept showing the main current paths for the $\mathbf{J}_{1}$ and $\mathbf{J}_{2}$ modes discussed above including the mirroring effect of the infinite ground plane. It could be simply stated that more opposing current paths lead to significant increase in $Q$.

We show detailed behaviour only for SAU2 (the situation is similar for SAU1) — see Figure 12 that confirms high $Q$ for the $\mathbf{J}_{1}$ mode. Characteristic angles are calculated for $H=$ $29 \mathrm{~mm}$, the actual height for which the dual-band antenna was designed [29].
5.1. The FCL-2 Fractal Antenna. The second presented structure is the so-called fractal clover leaf (FCL) of the second iteration, [14]. The antenna is fed by an L-probe [30] that excites its dominant mode and is located at height $H=$ $36 \mathrm{~mm}$. Actually, the dominant mode is composed of two degenerated modes $\mathbf{J}_{1}$ and $\mathbf{J}_{2}$ (Figure 13). The second higher mode $\mathbf{J}_{3}$ is shown at Figure 14 for completeness.

Figure 15 shows the main current paths of these modes, and we can again deduce that the dominant mode will exhibit 


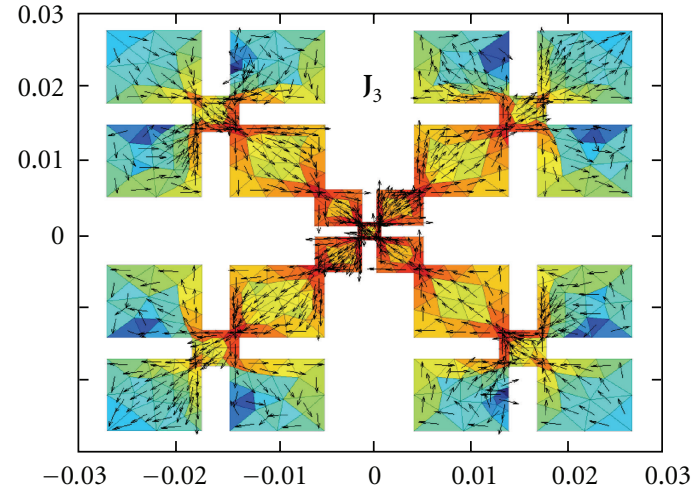

(a)

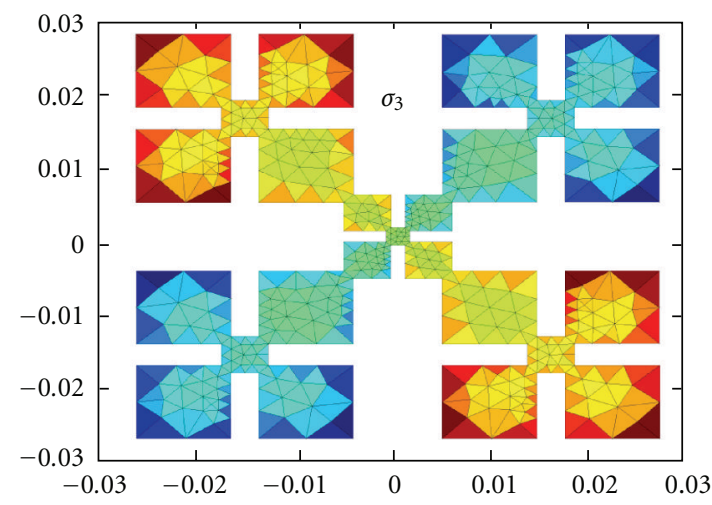

(b)

FIgURE 14: Second higher mode $\mathbf{J}_{3}$ (currents and charges).
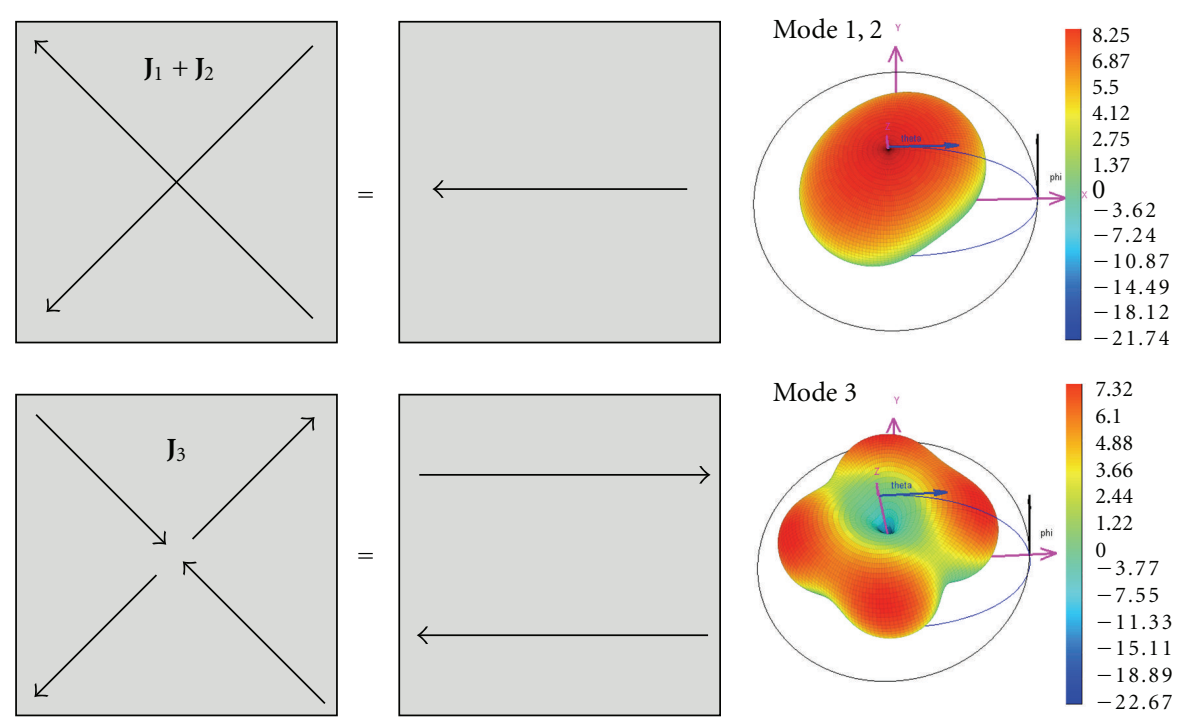

FIGURE 15: Schematic depiction of the dominant current paths for the dominant $\left(\mathbf{J}_{1}+\mathbf{J}_{2}\right)$ and the second higher $\mathbf{J}_{3}$ modes together with their modal radiation patterns.

lower $Q$ compared to $\mathbf{J}_{3}$. This is confirmed by Figure $16-\mathrm{J}_{3}$ has more than 200x higher radiation $Q$.

\section{Resonant Properties of Studied Antennas}

The properties of studied antennas are summarized in this section. At first we observed that microstrip antenna could support different kinds of modes regarding their $Q$ factors (see Figure 17):

(a) low $Q$ modes with the current flowing in one direction and not changing its phase (dominant modes of simple shapes like rectangular, circular patch, and so forth.)

(b) high $Q$ modes with part of the currents flowing in the opposite direction. These modes exist even on simple "U" shaped patch (Figure 9 left) and on complex (fractal) geometries.
Secondly, it has been observed that resonant frequency is quite a complicated function of height. Unfortunately we do not yet have any physical explanation as to why some modes present minimum values of $f_{r}$.

Looking at Figure 18, it is clear (and interesting) that the resonant frequency behaves quite differently for low$Q$ and high- $Q$ modes. The resonant frequency of low- $Q$ modes is much more sensitive to the height, whereas high- $Q$ modes exhibit almost constant $f_{r}$ when the height is varied. The proposed explanation is that the opposite currents (responsible for high $Q$ ) keep reactive fields very close to the radiating structure so the effect of a fringing field coupled to the ground plane becomes almost negligible.

\section{Conclusions}

Modal resonant properties of selected microstrip patch antennas have been studied with the help of characteristic 


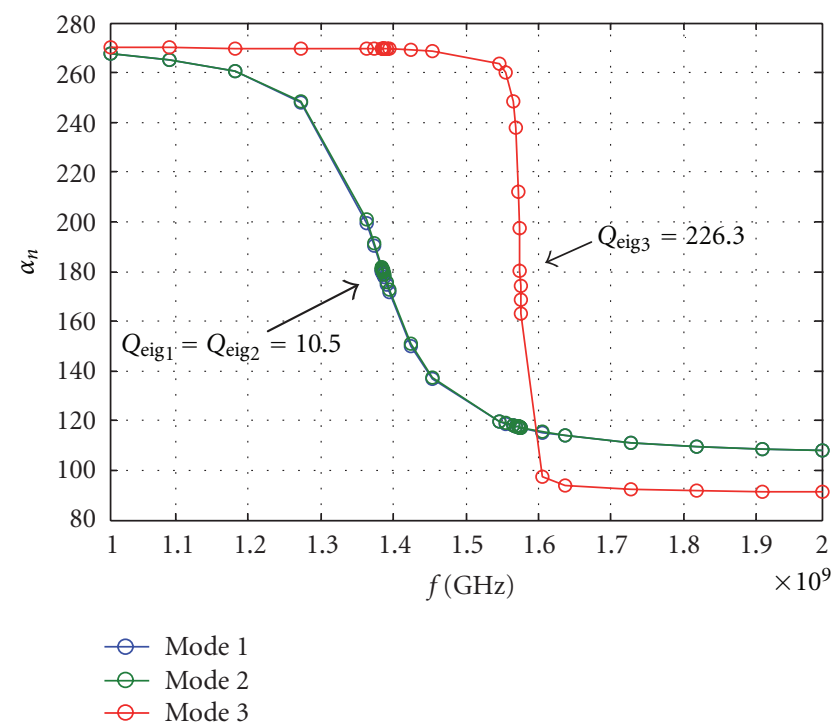

FIgURE 16: Characteristic angles for the FCL2 structure at $H=$ $29 \mathrm{~mm}$.

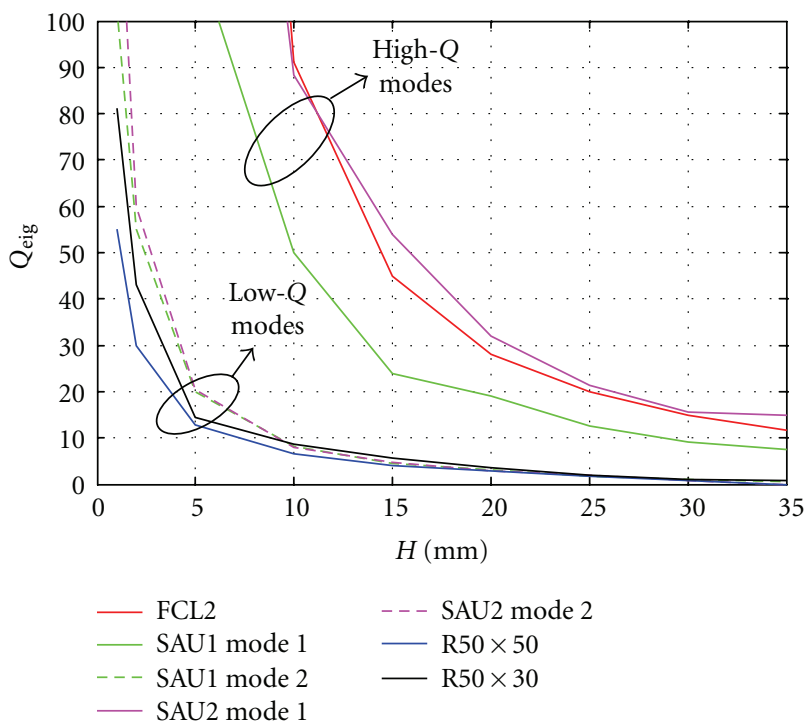

FIgURE 17: Radiation Qs for different antennas/modes.

modes and the novel theory published by Vandenbosch. It has been found that the resonant frequency of a simple rectangular patch antenna is quite a complicated function of its height above the infinite ground. Moreover, the dependency of resonant frequency is also found to be a function of the radiation $Q$ factor (which is now possible to calculate in a rigorous way). Due to the complexity of the problem, no physical explanation for the resonant frequency behaviour has yet been found.

It is observed that the radiation $Q$ factor decreases for "standard" heights $(<\sim 0.1 \lambda)$, however there exists an absolute minimum value of $Q$ that has already been predicted by simple modeling of two elementary out-of-phase dipoles.

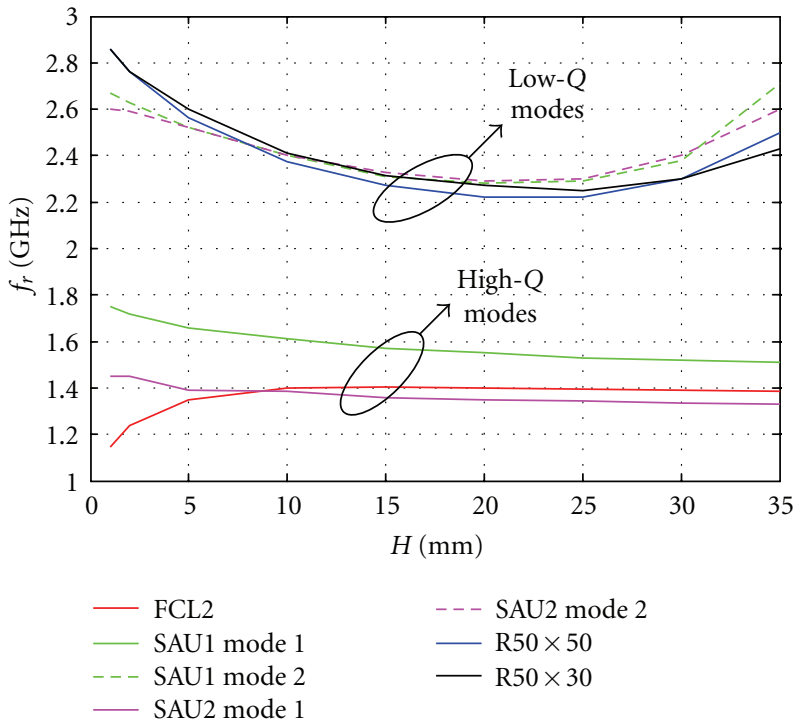

FIGURE 18: Resonant frequencies for different antennas/modes.

Using proper feeding techniques (like the L-probe) allows us to design wideband compact antennas.

The theory now puts current distribution and the radiation $Q$ factor into objective context. Whenever the current mode exhibits opposite components, high $Q$ may appear.

Future work is needed to connect the presented theory with parameter sweeps or even optimization, so we will be able to design novel wideband/multimode compact antennas.

\section{Acknowledgments}

This work was supported by the Grant Agency of the Czech Technical University in Prague, grant no. SGS11/065/ OHK3/1T/13 and by the Project COST 1102. The authors would like to thank professor Vandenbosch for fruitful discussions, Neil Bell for his comments, and two anonymous reviewers who suggested some improvements to the paper.

\section{References}

[1] P. Bhartia, I. Bahl, R. Garg, and A. Ittipiboon, Microstrip Antenna Design Handbook, Artech House, Norwood, Mass, USA, 2000.

[2] K. F. Lee and W. Chen, Advances in Microstrip and Printed Antennas, John Wiley \& Sons, New York, NY, USA, 1997.

[3] J. R. James, P. S. Hall, and C. Wood, Microstrip Antenna Theory and Design, Peter Peregrinus, London, UK, 1986.

[4] R. F. Harrington and J. Mautz, "Theory of characteristic modes for conducting bodies," IEEE Transactions on Antennas and Propagation, vol. 19, no. 5, pp. 622-628, 1971.

[5] E. A. Daviu, Analysis and design of antennas for wireless communications using modal methods, Ph.D. dissertation, Universidad Politécnica de Valencia, Valencia, Spain, 2008.

[6] G. A. E. Vandenbosch, "Reactive energies, impedance, and Q factor of radiating structures," IEEE Transactions on Antennas 
and Propagation, vol. 58, no. 4, Article ID 5398856, pp. 1112 1127, 2010.

[7] G. A. E. Vandenbosch and V. Volski, "Lower bounds for radiation Q of very small antennas of arbitrary topology," in Proceedings of the 4th European Conference on Antennas and Propagation (EuCAP '10), pp. 1-4, Barcelona, Spain, April 2010.

[8] R. F. Harrington, Field Calculation by the Method of Moments, IEEE Press, New York, NY, USA, 1993.

[9] M. Cabedo-Fabres, E. Antonino-Daviu, A. Valero-Nogueira, and M. F. Bataller, "The theory of characteristic modes revisited: a contribution to the design of antennas for modern applications," IEEE Antennas and Propagation Magazine, vol. 49, no. 5, pp. 52-68, 2007.

[10] P. Hazdra and P. Hamouz, "On the modal superposition lying under the MoM matrix equations," Radioengineering, vol. 17, no. 3, pp. 42-46, 2008.

[11] Mathworks, http://www.mathworks.com/.

[12] S. N. Makarov, Antenna and EM Modeling with Matlab, John Wiley \& Sons, New York, NY, USA, 2002.

[13] S. M. Rao, D. R. Wilton, and A. W. Glisson, "Electromagnetic scattering by surfaces of arbitrary shape," IEEE Transactions on Antennas and Propagation, vol. 30, no. 3, pp. 409-418, 1982.

[14] M. Capek, P. Hazdra, P. Hamouz, and M. Mazanek, "Software tools for efficient generation, modelling and optimisation of fractal radiating structures," IET Microwaves, Antennas and Propagation, vol. 5, no. 8, pp. 1002-1007, 2011.

[15] Comsol Multiphysics, http://www.comsol.com/.

[16] J. L. Volakis, Ch. Chen, and K. Fujimoto, Small Antennas: Miniaturization Techniques \& Applications, chapter 2, McGraw-Hill, New York, NY, USA, 1st edition, 2010.

[17] J. S. McLean, "A re-examination of the fundamental limits on the radiation q of electrically small antennas," IEEE Transactions on Antennas and Propagation, vol. 44, no. 5, pp. 672-676, 1996.

[18] R. F. Harrington and J. R. Mautz, "Control of radar scattering by reactive loading," IEEE Transactions on Antennas and Propagation, vol. 20, no. 4, pp. 446-454, 1972.

[19] R. F. Harrington, Time-Harmonic Electromagnetic Fields, IEEE Press, New York, NY, USA, 2001.

[20] D. R. Rhodes, "Observable stored energies of electromagnetic systems," Journal of the Franklin Institute, vol. 302, no. 3, pp. 225-237, 1976.

[21] D. R. Rhodes, "Reactance Theorem," Proceedings of the Royal Society A, vol. 353, no. 1672, pp. 1-10, 1977.

[22] J. Shaeffer, "MOM3D method of moments code theory manual," Research report 189594, Lockheed Advanced Development Company, 1992.

[23] M. Capek, P. Hazdra, and J. Eichler, "A method for the evaluation of radiation Q based on modal approach," IEEE Transactions on Antennas and Propagation. In press.

[24] P. Arcioni, M. Bressan, and L. Perregrini, "On the evaluation of the double surface integrals arising in the application of the boundary integral method to 3-D problems," IEEE Transactions on Microwave Theory and Techniques, vol. 45, no. 3, pp. 436-439, 1997.

[25] P. Hazdra, M. Capek, and J. Eichler, "Radiation Q-factors of thin-wire dipole arrangements," IEEE Antennas and Wireless Propagation Letters, vol. 10, pp. 556-560, 2011.

[26] A. D. Yaghjian and S. R. Best, "Impedance, bandwidth, and Q of antennas," IEEE Transactions on Antennas and Propagation, vol. 53, no. 4, pp. 1298-1324, 2005.

[27] CST Gmbh, http://www.cst.com/.
[28] S. N. Sinha and M. Jain, "A self-affine fractal multiband antenna," IEEE Antennas and Wireless Propagation Letters, vol. 6, Article ID 891519, pp. 110-112, 2007.

[29] J. Eichler, P. Hazdra, M. Čapek, T. Kořínek, and P. Hamouz, "Design of a dual-band orthogonally polarized l-probe-fed fractal patch antenna using modal methods," IEEE Antennas and Wireless Propagation Letters, vol. 10, pp. 1389-1392, 2011.

[30] C. L. Mak, K. M. Luk, K. F. Lee, and Y. L. Chow, "Experimental study of a microstrip patch antenna with an L-shaped probe," IEEE Transactions on Antennas and Propagation, vol. 48, no. 5, pp. 777-783, 2000. 

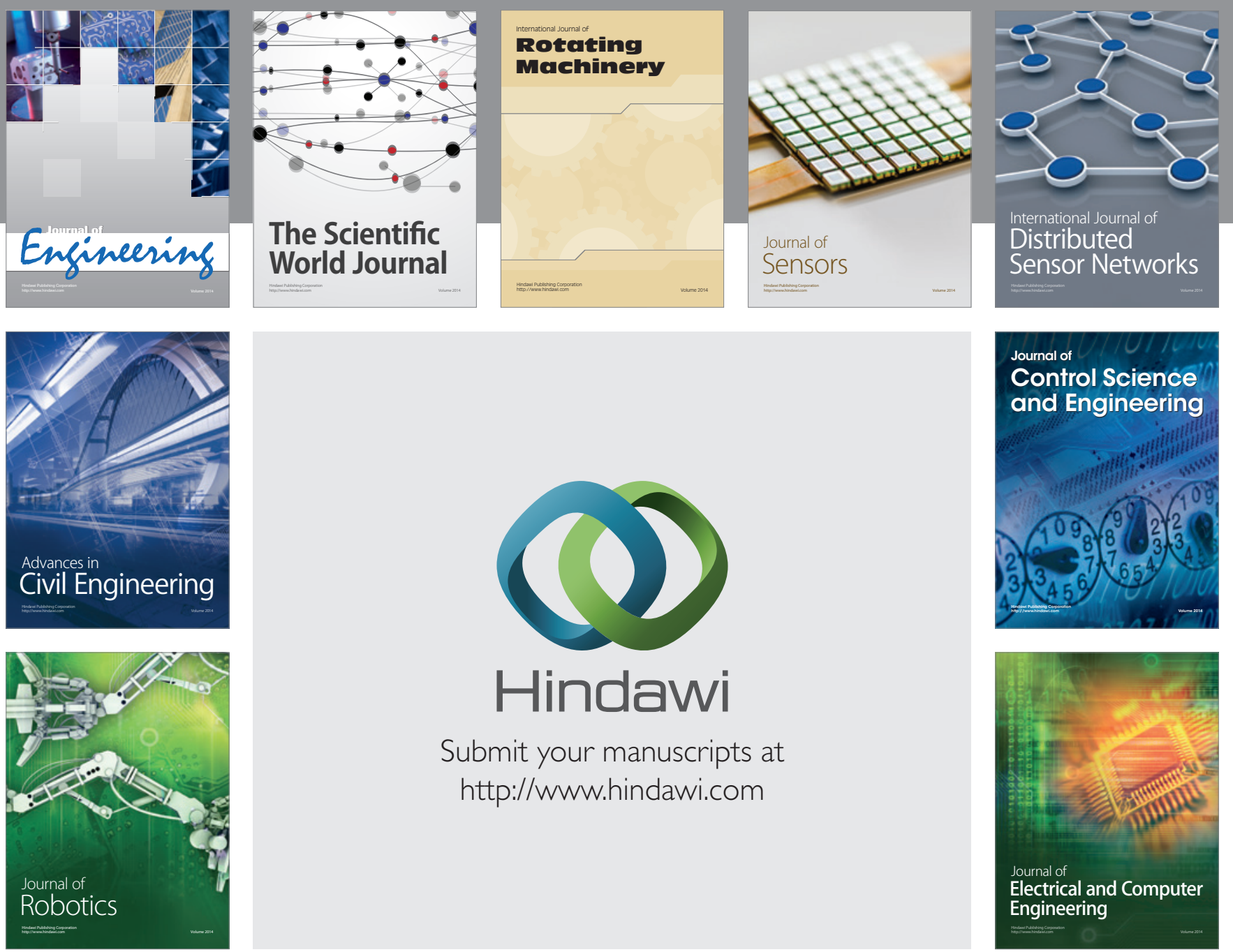

Submit your manuscripts at

http://www.hindawi.com
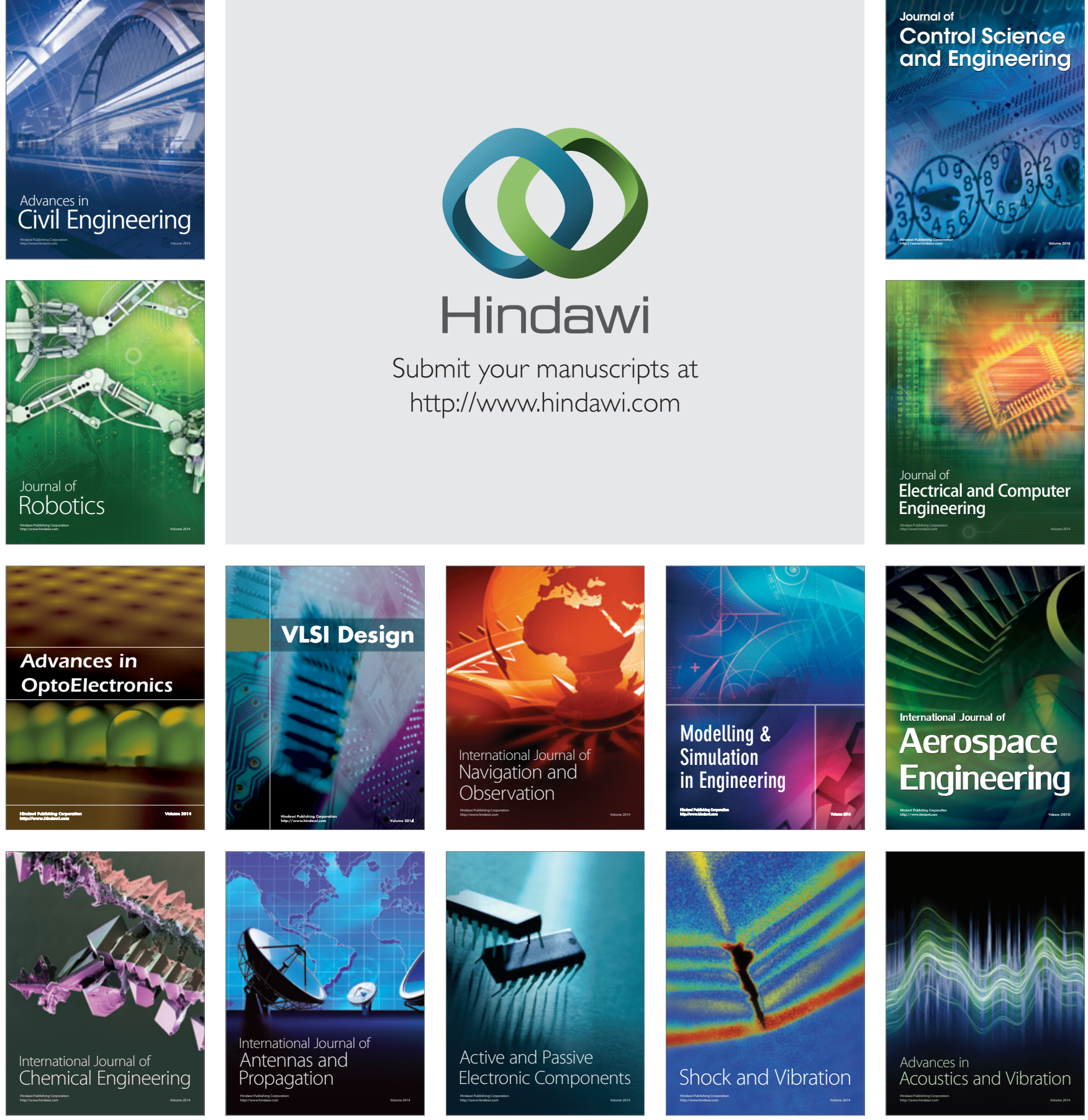